\title{
THE FOURIER ANALYSIS OF THE OBSERVED VELOCITY FIELD OF GAS IN THE INNER 1.5 PC OF THE GALAXY
}

A.M. FRIDMAN AND V.V. LYAKHOVICH

Institute of Astronomy of the Russian Academy of Science, 48, Pyatnitskaya St., Moscow, 109017, Russia

O.V. KHORUZHII

National Research Center "Troitsk Institute for Innovation and Thermonuclear Researches", Troitsk, Moscow reg., 142092, Russia

AND

O.K. SILCHENKO

Sternberg Astronomical Institute, Moscow State University, University prospect, 13, Moscow, 119899, Russia

\begin{abstract}
The Fourier analysis of the observed velocity field of ionized gas in the inner 1.5 pc of the Galactic Center (obtained by Roberts and Goss, 1993) is made. As follows from the analysis, the observed field of residual velocities is dominated by the second Fourier harmonic. This fact can be treated as a consequence of the presence of an one-armed density wave with the density maximum along the Northern Arm plus the Western Arc structure. The wave nature of this structure is proved on the base of the behaviour of the phase of the second harmonic of line-of-sight velocity field in the whole region. The Fourier analysis shows also the presence of systematic radial velocity. We consider this flow as a quasi-stationary radial drift caused by one-armed nonlinear density wave ('mini-spiral').
\end{abstract}

We investigate the dynamics of ionized gas in the inner $1.5 \mathrm{pc}$ of the Galaxy using a two-dimensional line-of-sight velocity field of the ionized gas obtained from a data cube (Roberts \& Goss, 1993) kindly provided to us by Dr. W. M. Goss and a new method based on the Fourier analysis of the velocity field (Lyakhovich et al., 1997.) The following results are obtained. 


\section{The presence of the component in pure rotation with a continuous one-armed spiral structure}

The line-of-sight velocity field obtained reminds that of a regularly rotating disk. Some areas are strongly deviate from the common regular rotation. However, the positions of the highest deviations from secular rotation agrees with the position of individual filaments and flows indicated in available literature (e.g. see Schwarz et al., 1989, Lacy et al., 1991).

Particularly, the Eastern Arm seems to lie outside the main gaseous disk and demonstrates strong positive velocity residuals. Moreover, this feature has an almost straight continuation to the west, and some signs of positive velocity residuals can be seen to the west from the Western Arc. Perhaps we deal with a large-scale gas flow off the gaseous disk plane.

The Northern Arm and the Western Arc tracing by emission brightness maximum are projected onto the area of near-zero velocity residuals and therefore may belong to a circularly rotating flat gaseous disk.

To investigate the dynamics of the inner gaseous disk, we have removed the elements related to the areas of strong non-circular motions. The subsequent analysis was made with this "rectified" velocity field.

Assuming an inclination of the central gaseous disk to be equal to $50^{\circ}$, P.A. $=215^{\circ}$ and attaching a dynamical center to $\operatorname{Sgr} A^{*}$, we have projected one-element rings onto the velocity field and have constructed azimuthal dependency of the line-of-sight velocity for a grid of radial distances ranged from $14 \operatorname{arcsec}$ to $42 \operatorname{arcsec}$ from $\operatorname{Sgr} A^{*}$.

A cosine-like form of these dependencies (Fig. 1) evidences for a domination of gas planar circular rotation inside $R \approx 1.7 \mathrm{pc}(42 \mathrm{arcsec})$.

\section{Evidences in favor of density wave nature of 'mini-spiral'}

¿From theoretical point of view (Lyakhovich et al., 1997), a line-of-sight velocity field of the gaseous disk where pure one-armed density wave is exited must consist of three Fourier harmonics only: zero, first, and second ones.

The Fourier analysis of the observed line-of-sight velocity field shows the real domination of these harmonics. The main component is the first cosine harmonic representing the circular rotation of the disk (about 100 $\mathrm{km} / \mathrm{s}$ ). Velocity residuals (zero and second harmonics) have amplitudes about $20 \mathrm{~km} / \mathrm{s}$.

For a tightly wound one-armed density wave we shown (Lyakhovich et $a l ., 1997)$ that the phase of the second harmonic of line-of-sight velocity, $F_{2}$, is shifted from the phase of the perturbed surface density approximately on $\pi / 2$ throughout the disk.

The close correlation of the one-armed spiral with the phase $\left(F_{2}-\pi / 2\right)$, that is the spiral described in the disk plane by the equation $\varphi(R)=$ 

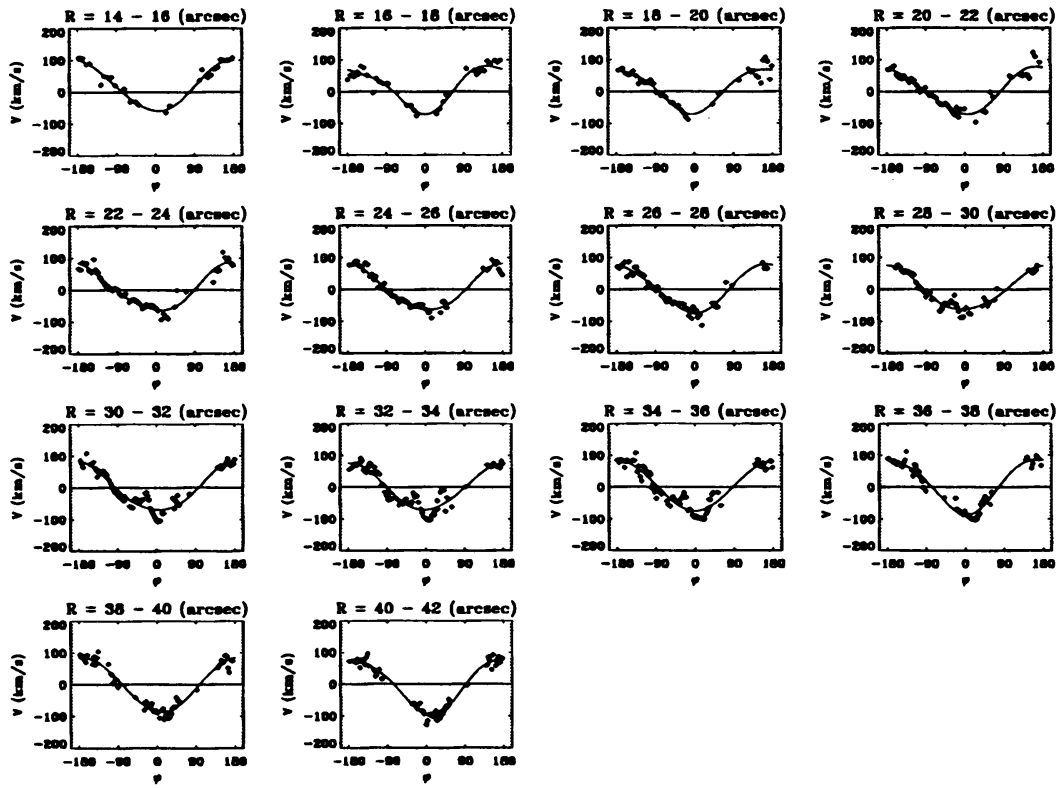

Figure 1. The azimuthal dependency of the line-of-sight velocity at different galactocentric radii in the disk plane. The velocities are averaged over ring with the width 2 ". The cosine-like form of these dependencies indicates domination of circular rotation of gas.

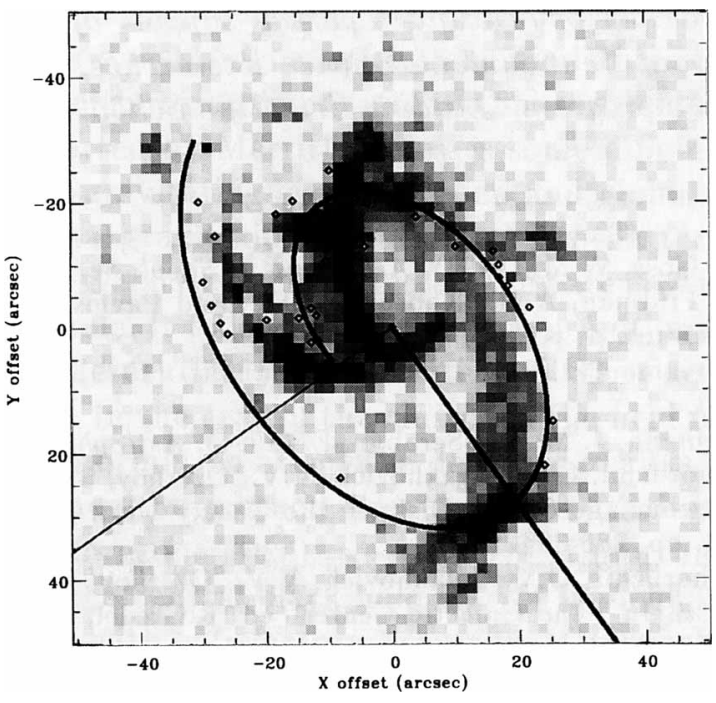

Figure 2. Similarity in shape of the one-armed spiral (diamonds) with phase $F_{2}-\pi / 2$, where $F_{2}$ is the phase of the second harmonic of line-of-sight velocity, and the image of the 'mini-spiral' (gray scale) proves the wave nature of the latter. The form of both spirals is close to the Archimedes spiral (solid line). 
$F_{2}(R)-\pi / 2$ where $F_{2}$ is the phase of the second harmonic of line-of-sight velocity, with form of Northern Arm and Western Arc proves the wave nature of the 'mini-spiral' (see Fig. 2). It is totally agree with our earlier results (Fridman et al., 1994, $1996 \mathrm{a}$, b), where it was shown that the system of the Northern Arm and the Western Arc is an one-armed trailing density wave generated by the over-reflection instability.

\section{Presence of the outflow in the disk}

The presence of the first sine harmonic in the observed line-of-sight velocity field deserves separate attention. The most reliable explanation of the observed feature is the presence of azimuthally symmetrical radial flows, accretion or outflow, in the disk. ¿From the observational data we conclude that the accretion takes place outside the inner $1.6 \mathrm{pc}$, and the outflow - inside.

The accretion can be explained in usual manner by the viscous transfer of momentum in the outer part of the disk. The mechanism of the slow outflow of the matter inside $1.6 \mathrm{pc}$ is an open question.

As the outflow region corresponds to the region with relatively strong density wave, it is natural to connect these phenomena with each other. Thus the outflow observed can be treated as a manifestation of the 'acoustic drift', the quasistationary radial flow of the disk material caused by a nonlinear density wave (Fridman \& Khoruzhii, 1994, Fridman et al., 1996).

This work was performed under partial support of RFBR grant $N$ 9602-17792, the grant "Fundamental Space Researches. Astronomy" for the 1997 year and the program "Leading Scientific Schools" grant 96-1596648.

\section{References}

Roberts, D.A. and Goss, W.M. (1993), Astrophys. J. Suppl. Ser., Vol. 86, p. 133.

Lyakhovich, V.V., Fridman, A.M., Khoruzhii, O.V. and Pavlov, A. (1997), Astronomy Reports, Vol. 41 no. 4, p. 447.

Schwarz, U. L., Bregman, J.D. and van Gorkom, J.H. (1989), Astron. G Astrophys., Vol. 215, p. 33.

Lacy, J. H., Achtermann, J. M. and Serabyn, E. (1991), Astrophys. J., Vol. 380, p. L71.

Fridman, A.M., Khoruzhii, O.V., Lyakhovich, V.V., Ozernoy, L. and Blitz L. (1994), In "Physics of Gaseous and Stellar Disks in the Galaxy", Ed. I.King, ASP Conference Series, Vol. 66, pp. 285-304.

Fridman, A.M., Khoruzhii, O.V., Lyakhovich, V.V., Ozernoy, L., Silchenko, O.K. and Blitz L. (1996 a), In "The Galactic Center" ed. R.Gredel, ASP Conference Series, Vol. 102, pp. 335-344.

Fridman, A.M., Khoruzhii, O.V., Lyakhovich, V.V., Ozernoy, L. and Blitz L. (1996 b), In "Bared Galaxies and Circumnuclear Activity", Nobel Symposium 98, Eds. Aa.Sandqvist and P.O.Lindblad, Springer-Verlag, pp. 193-200.

Fridman, A.M. and Khoruzhii, O.V. (1994), Appendix V to book A.M.Fridman and N.N.Gor'kavyi "Physics of Planetary Rings", 1994 (in Russian, english translation will appear in Springer in 1998).

Fridman, A.M., Khoruzhii, O.V. and Gor'kavyi N.N. (1996), Chaos, Vol. 6 no. 3, p. 334. 